\title{
La distinction des sexes dans l'au-delà médiéval
}

Jérôme Baschet

\section{(2) OpenEdition}

Journals

Édition électronique

URL : https://journals.openedition.org/clio/5363

DOI : 10.4000/clio.5363

ISSN : 1777-5299

Éditeur

Belin

Édition imprimée

Date de publication : 1 novembre 2007

Pagination : 17-36

ISBN : 978-2-85816-940-5

ISSN : 1252-7017

Référence électronique

Jérôme Baschet, "La distinction des sexes dans l'au-delà médiéval », Clio. Femmes, Genre, Histoire [En ligne], 26 | 2007, mis en ligne le 01 janvier 2010, consulté le 24 avril 2022. URL : http://

journals.openedition.org/clio/5363; DOI : https://doi.org/10.4000/clio.5363

Ce document a été généré automatiquement le 24 avril 2022.

Tous droits réservés 


\title{
La distinction des sexes dans l'au- delà médiéval
}

\author{
Jérôme Baschet
}

1 Au Moyen Âge, l'au-delà pèse d'un poids considérable sur l'ici-bas; et même si la vie terrestre acquiert une épaisseur de plus en plus assumée, la quête du salut dans l'autre monde en donne, finalement, le sens. Les lieux de l'au-delà, volontiers enclos, à l'image des murs de la Jérusalem céleste, instaurent les plus définitives des séparations. Après le Jugement dernier tout au moins, chacun est voué à demeurer éternellement dans le lieu qui correspond à ses fautes ou à ses mérites. Mais qu'en est-il alors de la distinction des sexes? A-t-elle sa place - son lieu - dans cette image d'une humanité passée au tamis de la justice divine? La question n'est pas sans importance, si l'on souligne que l'au-delà est le lieu de la vérité dévoilée, par opposition au monde d'ombres qu'est l'icibas: les deux sexes y sont-ils mêlés, associés, séparés? Leur distinction est-elle assumée, confortée par une différenciation de lieux, ou au contraire esquivée, effacée? Quelles significations ces options peuvent-elles avoir au sein des représentations propres à l'Occident médiéval ?

2 Ces interrogations invitent à croiser deux axes de réflexion : l'un concerne le statut de la distinction des sexes dans l'anthropologie chrétienne médiévale ; l'autre, l'évolution des représentations de l'au-delà. Sur ce second point, et puisque nous souhaitons nous interroger sur le marquage des lieux et la spécificité éventuelle des groupes, masculins ou féminins, qui les occupent, il est nécessaire d'insister sur le processus de localisation de l'au-delà qui se joue au cours du xile siècle. La naissance du purgatoire comme troisième lieu, mise en relief par Jacques Le Goff (en même temps que l'émergence des deux limbes), en est l'aspect le mieux connu. Elle contribue de façon importante à l'effort de l'Église pour fixer les âmes des défunts et parvenir à un «enfermement des morts »'. Plus encore qu'une " profonde réorganisation de la géographie de l'au-delà ", il s'agit sans doute de la naissance même d'une géographie de l'autre monde, car émerge alors la possibilité, théologiquement assumée, d'une représentation localisée du sort des âmes après la mort ${ }^{2}$. À partir de ce moment, un processus de distinction (de « discrétisation ») des lieux de l'au-delà est engagé. Ses premiers effets, repérables à la 
fin du xile siècle (naissance du purgatoire et des limbes), seront suivis par d'autres, notamment l'accentuation des subdivisions internes de l'enfer, qui se constituera comme système articulé de lieux de peines : les cercles infernaux décrits par Dante et les compartiments rocheux de l'enfer peints par Buonamico Buffalmacco au Camposanto de Pise, vers 1330-1340, étant deux expressions achevées de cette construction.

3 Ajoutons encore que ce processus de localisation de l'au-delà, puis de spécification de plus en plus fine des lieux qui le composent, doit être mis en rapport avec une logique sociale générale de spatialisation (ou pour être plus exact de localisation), qui est à l'œuvre dans l'Occident médiéval depuis le xie siècle et que le terme d'encellulement, forgé par Robert Fossier, synthétise avec force. Il n'est donc pas illégitime de se demander si ce processus permet ou non l'émergence de lieux spécifiques, clos ou non, venant séparer ou distinguer hommes et femmes. Mais, comme on l'a dit, cette question est inséparable d'une réflexion sur le statut de la distinction des sexes dans les représentations médiévales. On commencera donc par quelques indications sur cette question, puis, ouvrant les dossiers iconographiques qui nous concernent ici au premier chef, on analysera successivement l'iconographie dominante des xIe-XIIIe siècles, qui ne semble guère réserver des lieux distincts à chacun des deux sexes, puis l'émergence de la distinction des sexes à travers le modèle des chœurs des élus, et enfin les transformations des xive-xve siècles, qui permettent de lui faire une place croissante.

Sur la distinction des sexes dans l'anthropologie médiévale

Comme il est impossible d'évoquer cette question dans toute son ampleur, on se limitera aux aspects, très révélateurs, relatifs à la distinction des sexes dans l'au-delà ${ }^{3}$. Deux représentations contradictoires coexistent d'abord. La première tire parti des versets pauliniens (Gal 3, 28), en vertu desquels il n'y a, parmi les baptisés, devenus un dans le Christ, «ni esclave ni libre, ni homme ni femme ». Cette image s'applique tout particulièrement au paradis céleste, réalisation de l'Église triomphante et de l'unité du corps du Christ. Rappelons aussi que, s'agissant de l'au-delà médiéval, il faut garder à l'esprit une distinction très active entre l'au-delà présent, qui concerne les âmes au lendemain de la mort, et l'au-delà futur, après la résurrection des morts et le Jugement dernier, qui concerne la personne reformée dans l'unité de l'âme et du corps. Dans la mesure où, pour les théologiens médiévaux, il n'existe dans l'âme nulle distinction de genre, les représentations de l'au-delà présent pourraient privilégier un effacement de cette distinction. Il n'en va pourtant pas nécessairement ainsi, car, quoique noncorporelle, l'âme est dotée, selon la théorie augustinienne reprise au Moyen Âge, d'une « similitude de corps » (« similitudo corporis »), qui la relie d'autant mieux à l'identité de la personne dont elle a abandonné le corps. Rien n'interdit donc de lui attribuer, comme le font volontiers les images, l'apparence d'un corps sexué, sans contredire pour autant le fait qu'elle n'est marquée, dans son essence, par aucune distinction de genre.

5 S'agissant de l'au-delà futur, il faut rappeler le caractère fondamental de la doctrine de la résurrection des corps ${ }^{4}$. Mais comment concevoir les corps glorieux des élus, ces "corps spirituels" que nomme Paul (I Cor 15)? Les auteurs des premiers siècles, comme Origène ou Grégoire de Nysse, en proposent une lecture spiritualiste : les corps ressuscités se font esprits, se métamorphosent en corps éthérés, semblables aux anges. Les élus ressuscités seront donc dépourvus de caractères sexués, car l'humanité 
glorieuse du ciel ne peut qu'ignorer la distinction des sexes. Mais Augustin inverse la tendance et impose une interprétation matérielle des corps ressuscités, faits de la même chair que sur terre. L'expression de Paul - «corps spirituel »- devient alors un véritable oxymore : c'est sans cesser d'être fait de chair que le corps devient spirituel, en acquérant les qualités qui sont normalement celles de l'esprit (incorruptibilité, éternité, beauté parfaite, etc.). Il devient alors possible d'affirmer que les deux sexes ressuscitent, " parce que le sexe féminin n'est pas un défaut, mais un état naturel " $^{5}$. Parce qu'elle fait partie de l'œuvre du Créateur, la différence des sexes a pleinement sa place après la fin des Temps : "Celui qui institua les deux sexes, les restituera l'un et l'autre ». Mais si les corps glorieux des élus sont bien dotés de leurs organes génitaux respectifs, preuve que ceux-ci ne relèvent pas d'une corruption de la nature humaine, ils ne sauraient évidemment donner lieu à aucun exercice de la sexualité. Au cours du Moyen Âge, les théologiens ne font, à de très rares exceptions près, que conforter la doctrine augustinienne des corps glorieux des élus, conçus comme des corps sexués mais non sexuels. La réaffirmation de ces positions est particulièrement marquée au cours des xIIe -XIIIe siècles, face aux dissidences dualistes qui nient la résurrection des corps, en même temps qu'elles s'attaquent aux fondements du pouvoir sacerdotal et de l'institution ecclésiale.

6 Il est donc théologiquement admis que la distinction des sexes, voulue par Dieu, a sa place dans l'ordre parfait et éternel du Royaume céleste. Quant à l'au-delà immédiat des âmes, le cas est plus ambigu : il est possible d'y opter pour un effacement du genre (au titre de la part spirituelle de la personne, qui fonde l'homogénéité du genre humain) ou au contraire pour le marquage de celui-ci (au titre de la similitudo corporis des conceptions cléricales qui convergent, en cela, avec les représentations communes, portées à faire de l'âme un double de la personne vivante). Avant même de chercher à savoir ce qu'il peut en être d'une expression spatialisée de la distinction des sexes dans l'au-delà, la question consiste à se demander si les images de l'au-delà rendent ou non visibles la distinction des sexes.

L'indistinction paradisiaque des statuts et des sexes (XIe-XIIIe siècles)

7 C'est d'un au-delà à deux lieux, enfer et paradis, qu'il est question ici, car le purgatoire, comme le limbe des enfants, distingués théologiquement à partir de la fin du xiIe siècle, tardent à prendre forme dans l'iconographie ${ }^{6}$. Dans les représentations de l'enfer, la distinction des sexes est volontiers marquée. Durant cette période, l'enfer est surtout représenté comme un grouillement confus de corps enchevêtrés, à l'intérieur de la gueule monstrueuse et menaçante du Léviathan. Par exemple, dans une célèbre miniature du Psautier de Winchester, corps masculins et féminins se superposent, dans une promiscuité soulignant la charge sexuelle dont les représentations de l'enfer ne cesseront de jouer?. Dans la sculpture romane, les châtiments infernaux donnent souvent la vedette à l'avare, un homme avec sa bourse autour du cou, et à la luxurieuse, mordue aux seins et au sexe par des serpents. Même dans cette version minimale, réduite à deux figures, la dualité des sexes est nettement mise en évidence (et les signes de la féminité particulièrement visés). Mais les organes génitaux masculins ne sont pas toujours à l'abri : le portail de la Gloire à Saint-Jacques de Compostelle en montrait un exemplaire férocement mordu par un démon. Bref, les représentations de l'enfer accumulent les marques d'une sur-sexualisation angoissante, qui contribuent à construire l'image d'un lieu négatif et terrifiant. Elles font place largement, souvent avec emphase, à la distinction des sexes. 
Il en va tout autrement du côté paradisiaque. En Occident, jusqu'au xime siècle inclus, le paradis est représenté de façon dominante sous l'espèce du sein d'Abraham (en concurrence toutefois avec la Jérusalem céleste et le jardin paradisiaque). On montre alors les élus rassemblés dans le sein d'Abraham, à l'image du pauvre Lazare de la Parabole (Luc 16) et conformément à la liturgie funéraire, dont les formules demandent que l'âme du défunt accède au repos dans le sein du patriarche ${ }^{8}$. Cette représentation doit surtout son succès à sa grande force figurative : les œuvres les plus remarquables expriment la protection dont bénéficient les élus, en montrant leur union intime avec le patriarche et leur inclusion dans un tissu parfois presque indissociable de son corps. De plus, l'image donne à voir le paradis comme une réunion à une figure paternelle, Abraham, père de tous les fidèles selon l'esprit, qui protège affectueusement sa progéniture. Image idéale de la parenté spirituelle, la représentation du sein d'Abraham insiste sur la fraternité de tous les chrétiens harmonieusement rassemblés dans le sein du patriarche. Il en découle une forte homogénéisation des élus, qui fait disparaître toutes les marques distinctives de statut social, d'âge et de sexe. Selon les cas, ils sont tous masculins (d'une masculinité généralement euphémisée), ou bien ils montrent les traits d'une enfance qui efface toute affiliation de genre.

9 Serait-ce parce qu'il s'agit d'une représentation du séjour des âmes (et que le caractère non-sexué de celles-ci s'imposerait donc) ? Je ne le pense pas, car, s'il est vrai que la Parabole de Lazare associe le sein d'Abraham au sort présent des âmes, les théologiens médiévaux en ont considérablement amplifié la signification. Saint Thomas par exemple identifie sein d'Abraham et Royaume céleste. Et, de fait, il peut figurer le séjour des élus ressuscités dans les représentations du Jugement dernier, notamment aux portails des cathédrales gothiques. Le Jugement dernier de la cathédrale de Bourges (vers 1250) confirme de manière éclairante que l'effacement des distinctions de genre dans le sein d'Abraham n'est en aucune manière une négation du caractère sexué des corps ressuscités. Le tympan montre en effet la résurrection des morts, et les traits distinctifs des deux sexes y sont nettement visibles. Dans le cortège des élus (comme aussi dans celui des damnés), la distinction des sexes (et des statuts) est nette : des nobles femmes suivent un franciscain et un roi, en direction de la porte du ciel, où Pierre les accueille (fig. 1). Mais, aussitôt passé ce seuil, les élus se fondent dans le sein d'Abraham, tous semblables. L'accès à la béatitude céleste se donne à voir comme une puissante métamorphose, par laquelle les corps ressuscités et sexués redeviennent à la fois comme des enfants (en vertu de l'évocation évangélique de l'enfance, condition d'accès au royaume des cieux; Mt 18,3) et comme des âmes (en vertu de l'oxymore qui conçoit les corps glorieux comme des « corps spirituels»).

10 Le système qui prédomine alors oppose d'un côté l'enfer, marqué par une insistance sur la distinction des sexes, sous l'espèce d'une promiscuité charnelle fort inquiétante du fait de son association aux tourments éternels, et de l'autre le paradis où la béatitude des élus s'exprime par la fusion dans la communauté asexuée des germains spirituels. La distinction des sexes ne saurait donc recouper une distinction de lieux : d'un côté, la distinction des sexes est affichée, mais se donne à voir sous la forme d'un coupable enchevêtrement; de l'autre, prévaut l'indistinction des sexes ${ }^{10}$.

11 Quelques remarques permettront d'étoffer un peu cette esquisse. Il convient d'abord d'observer que les représentations de l'au-delà, aux xie- xIIe siècles, ne font souvent qu'une place très faible aux femmes. Considérons seulement, parmi d'autres exemples possibles, trois Jugements derniers. Celui de l'Apocalypse de Bamberg (fig. 3), manuscrit 
commandé par Otton III et réalisé à Reichenau ${ }^{11}$, ne montre aucune femme parmi les ressuscités, deux parmi les seize réprouvés (dont une clairement associée au souverain qui plonge le premier dans l'enfer) et une seule dans le cortège des élus, tout à fait à l'arrière-plan (sur douze figures). Au total, trois femmes pour trente-cinq personnages masculins (sans compter le Christ, les apôtres et saint Jean visionnaire). Le moins que l'on puisse dire est que, dans un grand monastère lié au pouvoir impérial comme l'était Reichenau, la représentation du Jugement dernier, événement cosmique qui récapitule la destinée de toute l'humanité, n'accorde qu'un intérêt bien marginal à la part féminine de celle-ci. Dans le Jugement dernier peint au revers de façade de l'abbatiale de Sant'Angelo in Formis, à la fin du xie siècle ${ }^{12}$, le cortège des élus est formé essentiellement de bénédictins, de prélats et de souverains, tandis que sept femmes presque toutes voilées (sur un total de trente-quatre figures) se serrent en queue de cortège, pour la plupart à peine visibles (fig. 4). Plus remarquable encore : l'assemblée des justes (non pas ici dans le sein d'Abraham, mais dans le jardin paradisiaque) forme une foule rythmée par la diversité des vêtements, mais homogène par les traits exclusivement masculins des figures. L'absence du féminin est d'autant plus remarquable que l'enfer, en vis-à-vis, accorde une forte visibilité à un couple hommefemme, lié par un même serpent. L'exclusion de la dualité des sexes, du côté paradisiaque, peut donc s'accomplir soit par une indistinction des sexes, soit par une représentation de l'humanité par sa seule part masculine.

Pour rester dans un milieu monastique (masculin), mentionnons le tympan de l'abbatiale Sainte-Foy de Conques, au début du xire siècle, qui montre l'un des Jugements derniers les plus développés de l'art roman ${ }^{13}$. En dépit de ce que l'on a dit de l'image romane du châtiment de la luxurieuse, l'ample enfer de Conques ne fait pas une place particulièrement forte aux femmes : parmi vingt-huit damnés qui y sont torturés, deux seulement sont des femmes (et toutes deux sont associées à un homme, l'une pour mettre en scène un couple luxurieux, l'autre la virago dominatrice) ${ }^{14}$. À tout prendre, les femmes sont plus à l'honneur du côté des élus : la Vierge, en tête du cortège, touche la mandorle du Christ, tandis que sainte Foy, patronne du lieu, est montrée dans sa fonction d'intercession. Surtout, la représentation du sein d'Abraham n'occupe que l'une des six arcades qui forment la "Jérusalem céleste d'Abraham ». Chaque arcade délimite ainsi un lieu spécifique où se tiennent deux figures, représentant à chaque fois une catégorie distincte d'élus. C'est l'une des occurrences précoces, dans l'art monumental, des chœurs des saints, dont on reparlera plus loin. Deux de ces lieux sont réservés aux femmes, l'un aux Vierges sages de la parabole évangélique (avec leurs lampes allumées), l'autre sans doute aux saintes Femmes (tenant des fioles de parfum). Cette fois, nous observons une distinction des lieux associée à la distinction des sexes, de surcroît sous un rapport relativement avantageux pour le féminin. L'importance de sainte Foy, vierge et martyre, objet du culte auquel l'abbaye doit son prestige, n'est sans doute pas étrangère à ce fait.

13 Nous sommes passés du côté des exceptions. On y ajoutera l'exemple du Jugement dernier provenant du monastère des bénédictines de Santa Maria in Campo Marzio, à Rome ${ }^{15}$ (fig. 2). Au registre inférieur, la Vierge et deux saintes occupent le centre de la Jérusalem céleste ; elles sont entourées de deux autres femmes, puis de cinq hommes de chaque côté; devant la muraille de la cité, figurent les deux donatrices, l'abbesse Constantia et Benedicta. Sept femmes sur un total de dix-sept figures associées au lieu paradisiaque. Le déséquilibre, faible, est de surcroît compensé par un avantage 
qualitatif: les femmes sont ici au centre et au premier plan. Du côté infernal, la figuration des damnés en groupes compacts n'autorise guère un décompte significatif, mais l'œuvre comporte, trait rare dans cette période, des inscriptions dénonçant divers péchés: des six encore lisibles, trois concernent spécifiquement les femmes (par exemple, mulier qui (sic!) in ecclesia locuta est). Au total, la production d'images, aux xie et xile siècles, est fortement liée aux milieux cléricaux et surtout aux branches masculines des bénédictins, ce qui favorise une élision du féminin (encore que ce résultat ne soit pas mécaniquement déterminé, puisque le rôle de la Vierge ou d'une sainte patronne peut modifier la donne ${ }^{16}$ ). Mais quelques œuvres plus rares, liées au monachisme féminin, semblent pouvoir restaurer partiellement l'équilibre (sans automatisme, là non plus). L'humanité dont l'image montre le sort dans l'au-delà peut retrouver la parité entre hommes et femmes. Mais, pour autant, nous n'avons guère rencontré encore de lieux spécifiquement associés à chacun des deux sexes.

Les premières représentations des chœurs des élus

Il faut maintenant faire place à la représentation des chœurs des élus. Dans ses premières apparitions iconographiques, elle ne concerne pas directement le Jugement dernier, ni le sort de l'humanité commune dans l'au-delà. Son objet est distinct de celui des œuvres commentées jusqu'à présent: il s'agit de figurer l'assemblée des saints rassemblés autour de la Majesté divine, dans leur rôle d'intercesseurs.

Les Pères de l'Église ont très tôt évoqué l'ordre convenant à la société céleste, dans laquelle les chœurs des saints viennent compléter les hiérarchies angéliques ${ }^{17}$. Mais c'est surtout avec l'essor de la liturgie que les chœurs des saints connaissent des mentions de plus en plus fréquentes. Ainsi, les formules des rituels de funérailles, dans les liturgies gallicanes et wisigothiques du haut Moyen Âge, demandent que l'âme rejoigne les chœurs des patriarches, des prophètes, des apôtres et des martyrs; et on y précise parfois la catégorie à laquelle l'âme du défunt est appelée à s'agréger, notamment pour les évêques (parmi les confesseurs) ou les vierges (qui rejoignent les vierges sages $)^{18}$. Dans les litanies, qui se diffusent en Occident au xe siècle, les saints intercesseurs sont également invoqués par catégories. Les six chœurs (chorus, exercitus ou numerus) des saints - patriarches, prophètes, apôtres, martyrs, confesseurs, vierges sont dès lors invoqués avec une grande fréquence dans la vie liturgique de l'Occident. À partir du xile siècle, cette mention des hiérarchies célestes est associée à de nombreux rituels, y compris le baptême ${ }^{19}$. L'imaginaire du monde des saints, consolidé par la liturgie et les prières, est donc fortement hiérarchisé et cet ordre conduit à séparer les deux sexes. Il existe une catégorie spécifiquement féminine (les vierges), les autres étant nettement masculines (apôtres, prophètes, confesseurs - catégorie qui tend à être identifiée au clergé) ; quant aux patriarches, ils incluent des femmes, à commencer par Ève, de même que les martyrs pourraient faire place aux femmes (mais les saintes martyres sont assimilées aux vierges). Bref, la sainteté féminine est-elle toute entière comprise dans la catégorie des vierges? Les images renforcent-elles ou non cette lecture des chœurs de saints?

L'une des premières figurations apparait, vers 925 , dans le psautier d'Aethelstan ${ }^{20}$. Deux pleines pages se complètent, représentant à chaque fois le Christ entouré de trois des chœurs célestes. Dans la seconde (fig. 5), le Christ apparaît au centre, dans une gloire en amande. Une deuxième gloire, de même forme mais plus ample, inclut les chœurs des martyrs (en haut), des confesseurs (au centre) et des vierges (en bas) ${ }^{21}$. Ils sont séparés par des bandes ornementales, également utilisées pour marquer l'axe vertical des deux 
mandorles, mais sans qu'on doive supposer une distinction signifiante au sein du chœur des vierges (ou de celui des martyrs). Même si les figures sont faiblement différenciées, les catégories de saints restent nettement identifiables (les confesseurs par la tonsure et parfois par le pallium; les vierges par le voile). Les vierges apparaissent sous la mandorle du Christ, à la place la moins éminente, conformément à l'ordre institué par les mentions liturgiques des chœurs des saints.

17 À la fin du xe siècle, les chœurs des saints apparaissent dans les Sacramentaires de Fulda, pour illustrer la fête de la Toussaint ${ }^{22}$. Dans un manuscrit enluminé par Ingelard à Saint-Germain-des-Prés, vers 1050-1060, confesseurs, martyrs et vierges forment trois groupes compacts sous la Majesté divine et la Vierge trônante ${ }^{23}$. Au début du XIIe siècle, il faut mentionner un manuscrit du commentaire d'Haymon d'Auxerre sur l'Apocalypse, où la hiérarchie des chœurs célestes figure deux fois ${ }^{24}$. Elle apparaît dans l'image de la Jérusalem céleste, mais aussi dans le Jugement dernier, qui la précède en vis-à-vis. L'intégration des chœurs des élus dans le Jugement dernier, synthétisant à la fois le collège des assesseurs et le cortège des élus, est une innovation remarquable, à peu près sans postérité immédiate. Dans le tiers inférieur de la page, à la droite du Christ, apparaissent ainsi, sur six registres superposés, les chœurs célestes : prophètes et patriarches sont réunis, tandis que les monachi sont dissociés des clercs séculiers, identifiés ici comme pontifices. Les vierges occupent le rang inférieur (les autres registres étant entièrement masculins). Sur la page suivante (fig. 7), les saints sont disposés autour de l'Agneau, dans une Jérusalem céleste circulaire. Ils se répartissent cette fois en cinq groupes, délimités par des bandes en quart de cercle : prophètes et patriarches ensemble, apôtres, confesseurs, martyrs, vierges. Curieusement, la "clôture " des vierges est imparfaitement dessinée et permet un contact - très spirituel, il est vrai - avec les apôtres. Cette fois, les vierges occupent une place de choix, au-dessus de l'Agneau et au centre, entre les apôtres et les prophètespatriarches. Cette réévaluation est liée à la présence d'une femme couronnée, de plus grande taille, au milieu des vierges : Marie sans doute ${ }^{25}$.

Au total, la représentation des chœurs des élus offre une vision nettement distincte de celle du sein d'Abraham. Au lieu de la communauté fusionnelle et indistincte des germains spirituels, elle montre une société céleste hiérarchisée, distinguant fortement les statuts. Mais ces deux types de représentations n'ont pas la même fonction : alors que le sein d'Abraham figure le sort des simples fidèles dans l'au-delà, les chœurs célestes présentent la parfaite société des saints intercesseurs, que l'on invoque, en bon ordre, dans la liturgie et la récitation fréquente des litanies. Quoi qu'il en soit, et bien que ce ne soit pas son objet direct, ce type de représentation conduit à distinguer les sexes. Distinction qui est même renforcée par l'image, puisque, sauf exception, le sixième chœur des élus est le seul qui figure des femmes, les cinq autres étant strictement masculins. Cette place restreinte s'avère assez conforme aux représentations d'une société dominée par la caste sacerdotale. Pour autant, la hiérarchie des chœurs des saints ne constitue pas un reflet de la société chrétienne, ni même de ses représentations : elle inclut des groupes d'un lointain passé (patriarches, prophètes, apôtres) et ignore bien des catégories importantes du temps (à commencer par les laïcs). Reste que le chœur des vierges joue un rôle important: il offre certainement un précieux référent céleste à la pratique du monachisme féminin (ce qui est d'autant plus probable que les usages des chœurs célestes retravaillent les catégories patristiques et liturgiques en fonction de l'importance acquise par le monde monastique, comme l'indique la division des confesseurs en pontifices et monachi). Mais 
cette contextualisation évolue au moment où la figuration de la cour céleste, encore rare à l'époque considérée jusqu'ici, se substitue à celle du sein d'Abraham.

Les transformations des XIVe-XVe siècles : ordre céleste et distinctions terrestres

$\mathrm{Au}$ xIve et surtout au xve siècle, l'image de la cour céleste, où les chœurs des saints s'ordonnent autour de la divinité, devient la figuration dominante du paradis. Le sein d'Abraham cède le pas, face au désir croissant d'évoquer la réunion directe des élus à Dieu et la vision béatifique, qui est l'essence de la récompense céleste ${ }^{26}$. Le sein fusionnel du patriarche fait place alors à la diversité hiérarchique de la cour céleste. Celle-ci se diffuse dans les représentations de la seule Jérusalem céleste, mais aussi dans les mises en image complètes des lieux de l'au-delà, comme celle que propose Enguerrand Quarton, en 1454, dans son Couronnement de la Vierge par La Trinité (enfer, purgatoire, limbe des enfants, paradis ; fig. 6). La cour céleste s'y répartit en huit groupes, disposés sur quatre registres (auquel il faut ajouter les saints Innocents): patriarches et prophètes; apôtres ; martyrs ; confesseurs (clercs séculiers); fondateurs d'ordres monastiques et mendiants ; vierges ; puis, au registre inférieur, les états du monde représentées d'un côté par les puissants (pape, cardinal, empereur, roi) et de l'autre par les simples laïcs. Ce dernier groupe est principalement masculin, mais des têtes féminines émergent au second plan. Coexistent donc, de manière fort cohérente, deux représentations des femmes: l'une séparée, dans le cas des vierges; l'autre associée, en position mineure, aux hommes, pour signifier la condition des laïcs. Un peu plus tard, la cour céleste figurée dans un missel d'Évreux donne une place plus significative aux femmes ${ }^{27}$. La foule des saints placés au premier plan, dans cette représentation qui joue habilement de la mise en perspective, est certes strictement masculine ; mais les femmes occupent le registre supérieur, plus étroit mais placé de part et d'autre de La Trinité et de la Vierge : à leur gauche, apparaissent les vierges à la tête voilée, à leur droite, un groupe de saintes non voilées. Le lieu propre des femmes est ici nettement mieux mis en valeur et fait l'objet d'une distinction fonctionnelle interne.

La place des femmes se modifie également dans le Jugement dernier, notamment dans la représentation du cortège des élus. Prenons l'exemple des fresques de Buonamico Buffalmacco au Camposanto de Pise $^{28}$. Selon une option anticipée par l'Apocalypse d'Oxford, citée précédemment, et qui va désormais faire école, le cortège des élus constitue en même temps une anticipation de la cour céleste ${ }^{29}$. Les élus y sont répartis en six rangées superposées (cinq et demie, en fait) : celle du haut commence avec les patriarches, Adam et Ève en tête; la suivante, menée par Jean Baptiste, inclut les confesseurs (notamment moines et ordres mendiants) ; la troisième est composée des dominants cléricaux et laïques; la quatrième de laïcs, bourgeois et hommes de loi. Ces quatre groupes, les plus éminents, sont entièrement masculins (à l'exception d'Ève) ; les deux suivants (un et demi, plutôt), moins éminents mais aussi plus proches du regard des spectateurs, sont strictement féminins : reines, nobles femmes, simples laïques et une moniale ${ }^{30}$. Le cortège des élus accorde donc une place spécifique, et séparée, aux femmes, en y mêlant différents statuts (laïcs surtout) et sans référence à la traditionnelle catégorie des vierges.

21 Comme d'autres œuvres du xve siècle, un retable du Maestro de Artés, actif à Valence vers 1500 , conjoint la représentation du Jugement dernier et celle du sort des âmes ${ }^{31}$ (fig. 8). Le Christ-Juge apparaît dans l'enceinte de la Jérusalem céleste. Mais au lieu d'être entouré des douze apôtres, comme il est normal, la traditionnelle polarité des 
sexes, marquée par l'intercession de Marie et de Jean, paraît s'être étendue à l'ensemble des occupants de la cité céleste : tandis que le Baptiste est accompagné de deux apôtres, Marie est associée à un groupe uniquement féminin (faisant fortement allusion aux saintes femmes de l'Évangile). Malgré le nombre restreint de figures (cinq femmes/ trois hommes), il est remarquable que la cité céleste, centrée sur le Christ, soit tout entière traversée par la distinction des sexes : les femmes d'un côté, les hommes de l'autre ${ }^{32}$ (à l'image de la disposition prévalant dans l'espace ecclésial, ainsi que dans les représentations de la Vierge au Manteau ${ }^{33}$ ). On ne saurait associer plus clairement distinction des sexes et distinction des lieux. Mais l'œuvre réserve d'autres surprises. Dans le purgatoire, combiné ici à la Messe de saint Grégoire, le caractère sexué des figures (des âmes dotées d'apparences très incarnées) est nettement marqué (comme il l'est dans le purgatoire que Jean Colombe intègre aux Très Riches Heures du duc de Berry). Hommes et femmes sont bien identifiables, et c'est une femme, aux longs cheveux, qui est libérée par l'ange, puis accueillie au paradis par saint Pierre. En revanche, dans les flammes de l'enfer, où le clergé est particulièrement à l'honneur, pas une femme n'apparaît... Même si les opérateurs du salut sont masculins (l'axe Christ - Grégoire le Grand), la reconnaissance céleste de la distinction des sexes s'accompagne d'un avantage concédé à la part féminine de la chrétienté.

Dernier exemple, plus surprenant encore : les fresques de Santa Maria in Piano à Loreto Aprutino (Abruzzes, vers 1420-1430) superposent Jugement dernier et jugement collectif des âmes ${ }^{34}$ (fig. 9). Au registre inférieur, le long cheminement des âmes, interrompu par l'épreuve du pont, les conduit jusqu'au palais et au jardin paradisiaques : ici, les élus, nus, laissent voir une nette dualité des sexes. En revanche, au registre supérieur, hormis les fondateurs des ordres mendiants en prière devant l'autel, l'assemblée céleste qui entoure le Juge, dans une atmosphère courtoise, égayée par une musique angélique, présente une étonnante répartition. Au centre, des saints (dont les apôtres) sont presque tous masculins, tandis que de part et d'autre, deux groupes amples montrent des élus non saints : il s'agit cette fois uniquement de femmes (les unes, voilées, semblent être des moniales; les autres sont élégamment vêtues). S'il est remarquable de constater que les femmes monopolisent ici la place des élus non saints, on prendra garde d'oublier la prééminence du groupe central des saints masculins. On pourra se demander si cette forte présence céleste des femmes est liée à un usage spécifique de l'édifice ou à une particularité de la commande des fresques ${ }^{35}$; en tout cas, elle radicalise singulièrement les tendances repérées dans les autres œuvres qu'on vient d'analyser.

C'est donc en considérant une double tension (distinction/indistinction; séparation ou conjonction spatiale) qu'il convient de s'interroger sur l'au-delà comme lieu de dévoilement des rapports de genre, tels que la volonté divine les inscrit à l'horizon de la fin des temps. Même si les observations avancées ici appellent une analyse plus systématique, le changement d'équilibre est frappant. On glisse d'une sousreprésentation féminine dans les lieux positifs de l'au-delà (place faible ou presque nulle parmi les élus) à une répartition nettement plus équilibrée, voire donnant l'avantage aux femmes. On verra là, sans trop de peine, l'effet du passage d'un art conçu par et pour les milieux cléricaux, et surtout monastiques, à un art certes tout autant inscrit dans l'ordre socio-ecclésial, mais selon des modalités transformées, faisant place 
à des interventions plus diversifiées, celles des autorités communales et des ordres mendiants notamment, et témoignant d'un souci désormais centré sur l'encadrement des laïcs, hommes et femmes.

Quelle place, au total, pour le féminin dans ce monde de la vérité révélée qu'est l'audelà ? Une place, tout d'abord, plutôt que rien, ce qui signifie une assomption positive de la polarité masculin/féminin. Côté infernal, prédominent l'accentuation des caractères sexués (des damnés et des diables) et une promiscuité dévalorisante entre les sexes, associée aux péchés et à leurs châtiments. Côté paradisiaque, s'affirment des lieux (ou tout au moins des groupes) propres, tirant parti de la vieille hiérarchie liturgique des chœurs célestes, pour en amplifier peu à peu l'usage. De la seule représentation des vierges, référent décisif pour les moniales (puisqu'il montre le lieu tant espéré, destination finale à laquelle doit conduire une vie de pénitence sous le régime de la clôture), on en vient à mettre en évidence des statuts féminins plus diversifiés, telles que ceux des veuves ou des laïques mariées.

Pour autant, on n'interprètera pas nécessairement ce phénomène comme une réhabilitation du féminin ou une féminisation du christianisme, conformément aux lectures qui fondent leur argumentation sur l'essor du culte marial, la diversification des formes de sainteté féminine ou l'amplification d'un symbolisme de la maternité nourricière ${ }^{36}$. Il me semble que le phénomène principal est moins l'attention croissante portée au féminin que le fait d'assumer progressivement, et positivement, la distinction des sexes. De manière générale, on fera l'hypothèse que celle-ci (avec ses hiérarchies constitutives, mais articulées à une dimension égalitaire) prend d'autant plus de relief que s'affirme la dynamique anti-dualiste qui traverse les siècles médiévaux, en rapport avec le statut de l'institution ecclésiale et son emprise croissante sur le monde. Pour ce qui nous concerne ici, un système de représentations, où la distinction des sexes est associée à la promiscuité infernale et à la dénonciation d'une chair coupable, est remplacé par un autre, qui fait place, dans les lieux positifs de l'au-delà, à la distinction des sexes. On parvient donc, en interaction manifeste avec les transformations sociales qui animent l'Occident des derniers siècles du Moyen Âge, à assumer la dualité des sexes comme caractéristique de l'image idéale de l'humanité que le lieu paradisiaque permet de construire. Pour cela, il aura fallu faire céder un autre complexe de représentations, dominant au cours des xie-XIIIe siècles, où le paradis était conçu comme l'image parfaite de la communauté fraternelle des chrétiens, rassemblée dans l'indistinction des sexes et des statuts, afin de pouvoir assumer pleinement ce que l'anthropologie augustinienne avait déjà établi, et donner ainsi corps, dans l'image paradisiaque de l'humanité réconciliée, à la distinction des sexes.

\section{BIBLIOGRAPHIE}

AUGUSTIN (saint), 1960, La Cité de Dieu, éd. B. Dombart et A. Kalb, Paris, Desclée (Bibliothèque Augustinienne, 37). 
BASCHET Jérôme, 1993, Les justices de l'au-delà. Les représentations de l'enfer en France et en Italie (xiiexve siècle), Rome, École française de Rome (Bibliothèque des Ecoles françaises d'Athènes et de Rome, 279).

-, 1995, « Jugement de l'âme, Jugement dernier : contradiction, complémentarité, chevauchement ?», Revue Mabillon, n. s., 6, p. 159-203.

-, 2000, Le sein du père. Abraham et la paternité dans l'Occident médiéval, Paris, Gallimard.

-, 2002, « I mondi del Medioevo : i luoghi dell'aldilà », in Enrico Castelnuovo et Giuseppe Sergi (dir.), Arti e Storia nel Mediovo. Volume primo, Tempi, spazi, istituzioni, Turin, Einaudi, p. 317-347.

-, 2006 (3e éd. mise à jour), La civilisation féodale. De l'an mil à la colonisation de l'Amérique, Paris, Champs-Flammarion.

-, sous presse, « Distinction des sexes et dualité de la personne dans les conceptions anthropologiques de l'Occident médiéval », in Irène Théry (dir.), Les aspects sexués de la vie sociale, Paris, Éditions de l'École des Hautes Études en Sciences Sociales (« Enquête »).

-, à paraître, « Une image à deux temps : Jugement dernier et jugement des âmes dans l'Occident médiéval », in Giovanni Careri, François Lissarague, Jean-Claude Schmitt, Carlo Severi (dir.), Tradition et temporalité des images.

BONNE Jean-Claude, 1984, L'art roman de face et de profil. Le tympan de Conques, Paris, Le Sycomore.

BYNUM Caroline, 1982, Jesus as mother. Studies in the Spirituality of the High Middle Ages, Berkeley, California University Press.

-, 1995, The Resurrection of the Body in Western Christianity, 200-1336, New York, Columbia University Press.

DONADIEU-RIGAUT, Dominique, 2005, Penser en images les ordres religieux (xiie-xve siècles), Paris, Arguments.

HENNING John, 1963-1964, « Die Chöre der Heiligen », Archiv für Liturgiewissenschaft, 8/2, p. 436-456.

IOGNA-PRAT Dominique, 1999, « Ordre(s) », in Jacques Le Goff et Jean-Claude Schmitt (dir.), Dictionnaire raisonné de l'Occident médiéval, Paris, Fayard, p. 845-860.

KLAPISCH-ZUBER Christiane, 1999, « Masculin/féminin », in Jacques Le Goff et Jean-Claude Schmitt (dir.), Dictionnaire raisonné de l'Occident médiéval, Paris, Fayard, p. 655-668.

LE GOFF Jacques, 1981, La naissance du Purgatoire, Paris, Gallimard.

-, 1986, « Les limbes », Nouvelle Revue de Psychanalyse, 34, p. 151-173.

NTEDIKA Joseph, 1971, L'évocation de l'au-delà dans la prière pour les morts. Étude de patristique et de liturgie latines (ive-viiie siècle), Louvain-Paris, Nauwelaerts.

PALAZZO Éric, 1994, Les sacramentaires de Fulda. Études sur l'iconographie et la liturgie à l'époque ottonienne, Münster, Aschendorff.

SCHMITT Jean-Claude, 1994, Les revenants. Les vivants et les morts dans la société médiévale, Paris, Gallimard.

\section{NOTES}

1.Le Goff 1981 et 1986 ; Schmitt 1994. 
2.Citation dans Le Goff 1981 : 14. Cette argumentation est développée dans Baschet 2002.

3.Pour une vision plus générale de cette question, je me permets de renvoyer à Baschet, sous presse. Concernant l'usage de l'expression « distinction des sexes », voir Cécile Barraud, « De la distinction de sexe dans les sociétés. Une présentation », dans Catherine Alès et Cécile Barraud (dir.), Sexe relatif ou sexe absolu ?, Paris, Ed. de la Maison des Sciences de l'Homme, 2001, p. 23-99.

4.Bynum 1995.

5.Augustin, Cité de Dieu, XXII, 17 (1960, 622).

6.Le limbe des patriarches fait l'objet de représentations abondantes et anciennes ; mais il s'agit d'un au-delà passé, d'un lieu vide depuis que le Christ y est descendu pour libérer les Justes, morts avant la Rédemption. Les représentations de la Descente du Christ aux limbes font clairement place à la distinction des sexes, puisque figurent, en tête du cortège des justes libérés, Adam et Ève (il s'agit pourtant d'âmes).

7.Pour tout ceci, Baschet 1993 (pl. II : Londres, British Library, Cott. Nero, C. IV, f. 39). 8.Baschet 2000 .

9.Voir le cahier d'images au milieu du volume.

10.Il y a bien sûr quelques exceptions, mais on ne peut en faire état ici. Il faut plutôt faire mention d'une autre représentation paradisiaque, peu fréquente mais significative : la « Jérusalem céleste alvéolaire » (Baschet 2000 : 241-243). Elle montre une architecture percée d'ouvertures avec, dans chacune d'elles, un élu en buste. L'option parait inverse de celle du sein d'Abraham : au lieu de la fusion communautaire en un lieu unique, chaque élu dispose de son lieu propre, de sa propre « cellule». Serait-ce l'image parfaite de l'enfermement local, de la clôture ? En fait, ce type d'image répond aux principes de la représentation romane : compartimentage, lien étroit entre la figure et son lieu. Bien qu'isolée, chaque figure ne fait que s'inscrire, comme les autres, dans une structure unique, qui forme le mur de la cité paradisiaque (selon l'équivalence, liturgiquement très active, entre les élus et les pierres de la Jérusalem céleste). De plus, malgré l'alternance régulière des couleurs des vêtements, prévaut l'uniformisation des élus, tous masculins, ou bien ramenés à un genre neutre.

11.Bamberg, Staatsbibliothek, Msc. Bibl. 140, f. 53 (début du xie siècle).

12.Baschet 1993 : 198-202, fig. 38-39. La réalisation des fresques a été engagée par l'abbé Desiderius du Mont-Cassin († 1087).

13.Bonne 1984 : 236-243.

14.L'enfer de l'Hortus Deliciarum, œuvre de l'abbesse Herrade de Landsberg, ne comporte que 4 femmes (infanticides et luxurieuses) sur un total de 24 damnés (Baschet 1993 : fig. 31).

15.Baschet 1993 : 195-198 et fig. 36-37. La datation de ce panneau (aujourd'hui à la Pinacothèque vaticane) a été très controversée (entre 1060-1070 et la première moitié du xiIIe siècle).

16.Il conviendrait de procéder à une comparaison précise entre les ordres religieux, en considérant notamment le cas des cisterciens, dont on sait qu'ils ont fait un usage abondant du symbolisme féminin (Bynum 1982).

17.Saint Cyprien mentionne patriarches, prophètes, apôtres, martyrs et vierges (Augustin évoque patriarches, prophètes, apôtres, martyrs et boni fideles), voir Ntedika 1971 : 220-225. Sur les notions d'ordre et de hiérarchie, Iogna-Prat 1999. 18.Ntedika 1971, notamment les formules du Gelasianum, 1646 et 1648-1656. 
19.Hennig 1963-1964 mentionne de nombreuses formules, par exemple la commendatio animae du XIIe siècle : " in nomine angelorum et archangelorum, in nomine patriarcharum et prophetarum, in nomine apostolorum et evangelistarum, in nomine martyrum et confessorum, in nomine sanctorum monachorum, in nomine sanctarum virginum et omnium sanctorum et sanctarum Dei ».

20.L'une des très rares occurrences antérieures figure dans le Sacramentaire de Metz, vers 870 (Paris, BNF, lat. 1141, f. 5v.-6) : anges, apôtres, martyrs, confesseurs et vierges en adoration devant la majesté divine.

21.Londres, British Library, Cotton Galba A XVIII, f. 21. L'autre page, au f. 2 v., est ordonnée en registres superposés : anges ; patriarches ; prophètes ; apôtres, avec la Vierge au milieu.

22.Palazzo, 1994.

23.Paris, BNF, lat. $11751, \mathrm{f}$. 1. On citera également la peinture murale de la Jérusalem céleste, à Saint-Chef-en-Dauphiné, où saintes femmes et moniales sont représentées à part (seconde moitié du xie siècle).

24.Oxford, Bodleian Library, Bod. 352, f. 12v. et 13 (manuscrit produit dans le sud-ouest de l'Allemagne).

25.Le lien entre Marie et les apôtres pourrait contribuer à rendre compte de la nonséparation des deux groupes. Au début du XIIe siècle, le chœur des vierges apparaît également dans le Psautier de Zwiefalten (Stuttgart, WLB, Brev. 100) et dans la Cité de Dieu de Florence (Bibl. Laurenziana, Plut. XII 17, f. 2 v.), où les six chœurs sont figurés dans leur version canonique, sans présence féminine hors du chœur des vierges.

26. Baschet 2000.

27.Évreux, Bibl. Mun., 99, f. 90 (Rouen, fin xve siècle ; Baschet 1993 : 427, pl. VII). Un autre exemple réduit les chœurs des saints à quatre groupes, ce qui augmente la place relative des vierges (Paris, Arsenal, 5204, f. 197, Mélanges, vers 1490). Les peintures murales de Santa Maria dei Bisognosi (Pereto, Abruzzes, 1488 ; Baschet 1993, p. 660-661, fig. 112-113) multiplient les groupes, identifiés par des inscriptions. Les femmes forment trois d'entre eux (vierges, veuves et martyres) et leur présence visuelle, importante, peut être estimée à un quart de l'ensemble. En dessous, l'enfer, insistant sur le châtiment des métiers, fait une place très réduite aux femmes : sur une trentaine de figures, deux sont clairement féminines.

28. Baschet 1993 : chapitre $v$ (phénomènes comparables dans les Jugements derniers italiens de cette période, notamment ceux de Giotto à Padoue, de Santa Maria Novella à Florence et de Tuscania).

29.Voir note 24 .

30.Le cortège des damnés présente une disposition assez comparable, à ceci près que, dans les trois rangées inférieures, à dominante féminine, les deux sexes sont mêlés. Dans l'enfer, hommes et femmes sont associés dans chacun des lieux de châtiments : la constitution de l'enfer comme système structuré de lieux ne s'accompagne d'aucune séparation spatiale entre hommes et femmes.

31.Sur ce type d'œuvres, Baschet, à paraître. On ignore malheureusement la destination initiale du retable.

32. Ce dispositif se diffuse à la fin du xve siècle : par exemple, Missel de Poitiers, Paris, BNF, lat. 873, f. 164 (Baschet 1993 : 417, pl. VI). Le Christ-Juge est entouré par deux groupes fournis : Marie et des saintes à sa droite ; les deux Jean et des saints à sa gauche. Sur terre, hommes et femmes soumis au Jugement sont distingués par la teinte 
de la peau (blanche/brune), ce qui souligne le caractère pleinement sexué des corps ressuscités.

33.La société des vivants est souvent disposée, sous le manteau de la Vierge, selon une distinction hommes (à sa droite)/femmes (à sa gauche). Quelques œuvres, reprenant la vision de Césaire de Heisterbach, montrent l'ordre cistercien inclus au ciel dans le manteau marial, avec les moines à la droite de la Vierge, les moniales à sa gauche (notamment retable de Jean Bellegambe, Musée de la Chartreuse de Douai) ; DonadieuRigaut 2005 : 60-66, fig. 19, 23.

34.Baschet $1995:$ 186-189.

35.Une inscription, dont seul le début reste lisible, paraît référer la fresque du Jugement à la contribution de la communauté paroissiale (Hoc opus fu(it) fact(um) de elimosina terre Laureti...). Loreto était placé sous la dépendance du comte Francesco II d'Aquino, tandis que Jeanne II d'Anjou a concédé, en 1429, une foire annuelle devant se dérouler devant l'église.

36.Je fais principalement allusion à Bynum 1982 et 1995.

\section{RÉSUMÉS}

La distinction des sexes dans l'au-delà médiéval. On s'efforce ici de préciser dans quelle mesure la dualité des sexes, assumée positivement par l'anthropologie augustinienne, trouve une expression spatialisée dans les représentations de l'au-delà, ce monde entièrement ordonné par la justice divine : la distinction des sexes s'exprime-t-elle par une distinction de lieux ? Aux XIXIIIe siècles, la distinction des sexes n'a guère de place que dans la coupable promiscuité de l'enfer, tandis que le paradis propose une image idéale de la fraternité spirituelle des baptisés, qui efface les distinctions de statuts et de sexes. Puis, l'ancienne hiérarchie patristique et liturgique des choeurs des élus se fraie un chemin dans l'image et sert d'appui à une amplification de la place spécifique des femmes dans les lieux paradisiaques. Enfin, dans la représentation ordonnée et hiérarchisée de la cour céleste, image dominante du paradis aux XIVe et XVe siècles, la dualité des sexes est désormais assumée positivement et mise en évidence visuellement comme l'un des aspects inhérents à l'humanité réconciliée.

The Gendering of the sexes in the medieval hereafter. This article seeks to understand how the difference between the sexes, which Augustianian theology recognized, was represented in medieval images of the hereafter. In this site of divine order were gender differences represented through spatial distinctions? Between the XIth and XIIIth centuries, differences between the sexes only emerged in images of hell, while representations of paradise expressed a vision of Christian spiritual brotherhood where social and sex differences were abolished. Progressively, the patristic and liturgical hierarchy of the choir of the elect gained weight in pictural representations thus contributing to establishing a specific place for women in heaven. Finally, during the XIVth and XVth centuries, the dominant image of paradise as an ordered and hierarchical celestial court, represented differences between the sexes in a positive fashion, as the visual evidence of humanity's reconciliation. 


\section{INDEX}

Mots-clés : Au-delà, enfer/paradis, Jugement dernier, masculin/féminin, société médiévale, genre

Keywords : Final Judgement, gender, hell/paradise, Hereafter, masculine/feminine, medieval society

\section{AUTEUR}

\section{JÉRÔME BASCHET}

Jérôme BASCHET est maître de conférences à l'EHESS, où il poursuit ses recherches et son enseignement sur l'anthropologie historique des images médiévales. Depuis 1997, il enseigne également à l'Universidad Autónoma de Chiapas (Mexique). Il est notamment l'auteur de Le sein du père. Abraham et la paternité dans l'Occident médiéval (Paris, Gallimard, 2000) et de La civilisation féodale. De l'an mil à la colonisation de l'Amérique (Paris, Flammarion, 2004 ; réédition 2006). 